\title{
ON THE ESSENTIAL SPECTRUM OF BANACH-SPACE OPERATORS
}

\author{
JÖRG ESCHMEIER \\ Fachbereich Mathematik, Universität des Saarlandes, D-66123 Saarbrücken, Germany
}

(Received 15 November 1998)

\begin{abstract}
Let $T$ and $S$ be quasisimilar operators on a Banach space $X$. A well-known result of Herrero shows that each component of the essential spectrum of $T$ meets the essential spectrum of $S$. Herrero used that, for an n-multicyclic operator, the components of the essential resolvent set with maximal negative index are simply connected. We give new and conceptually simpler proofs for both of Herrero's results based on the observation that on the essential resolvent set of $T$ the section spaces of the sheaves

$$
\operatorname{Ker}\left(\mathcal{O}_{\mathbb{C}}^{\prime} \hat{\otimes} X \stackrel{z-T}{\longrightarrow} \mathcal{O}_{\mathbb{C}}^{\prime} \hat{\otimes} X\right) \text { and } \operatorname{Coker}\left(\mathcal{O}_{\mathbb{C}} \hat{\otimes} X^{\prime} \stackrel{z-T^{\prime}}{\longrightarrow} \mathcal{O}_{\mathbb{C}} \hat{\otimes} X^{\prime}\right)
$$

are complete nuclear spaces that are topologically dual to each other. Other concrete applications of this result are given.
\end{abstract}

Keywords: quasisimilarity; essential spectrum; coherent sheaves; sheaf models

AMS 1991 Mathematics subject classification: Primary 47A53; 46A11

\section{Introduction}

Let $T \in L(X)$ be a continuous linear operator on a complex Banach space $X$. It is well known that the restrictions of the sheaves

$$
\operatorname{Ker}\left(\mathcal{O}_{\mathbb{C}}^{X} \stackrel{z-T}{\longrightarrow} \mathcal{O}_{\mathbb{C}}^{X}\right) \text { and } \operatorname{Coker}\left(\mathcal{O}_{\mathbb{C}}^{X} \stackrel{z-T}{\longrightarrow} \mathcal{O}_{\mathbb{C}}^{X}\right)
$$

to the essential resolvent set $\rho_{\mathrm{e}}(T)$ of $T$ are coherent analytic sheaves. This observation has been used by Putinar [12] to show that quasisimilar Banach-space operators with Bishop's property $(\beta)$ possess equal essential spectra. Let $T \in L(X)$ and $S \in L(Y)$ be quasisimilar operators on Banach spaces $X$ and $Y$. In [7], Herrero proved that each component of the essential spectrum of $T$ meets the essential spectrum of $S$, and vice versa. The proof given by Herrero is based on one of his earlier results on the Fredholm structure of multicyclic operators. More precisely, let $T$ be an $n$-multicyclic operator on a Banach space $X$. In [6], Herrero proved that every component of $\rho_{\mathrm{e}}(T)$ of index $-n$ is simply connected, and this is the main ingredient of the proof of the above cited result from [7].

In this paper we give extensions of Putinar's results, and we show that the same coherence principle can be used to obtain new proofs of both results of Herrero. More 
precisely, using the coherence of the above sheaves, one can show that the multiplication operator

$$
\mathcal{O}(U, X) \rightarrow \mathcal{O}(U, X), \quad f \mapsto(z-T) f
$$

has closed range for each open subset $U$ of $\rho_{\mathrm{e}}(T)$, and that the quotient space $\mathcal{F}_{T}(U)=$ $\mathcal{O}(U, X) /(z-T) \mathcal{O}(U, X)$ is a nuclear Fréchet space. Let $T^{\prime} \in L\left(X^{\prime}\right)$ be the adjoint of the operator $T$. We show that, for each open set $U \subset \rho_{\mathrm{e}}(T)$, the range of the operator $\mathcal{O}\left(U, X^{\prime}\right) \stackrel{z-T^{\prime}}{\longrightarrow} \mathcal{O}\left(U, X^{\prime}\right)$ is $w^{*}$-closed; that is, the range is weakly closed relative to the duality $\left\langle\mathcal{O}(U)^{\prime} \hat{\otimes} X, \mathcal{O}\left(U, X^{\prime}\right)\right\rangle$. Furthermore, the nuclear Fréchet space

$$
\mathcal{F}_{T^{\prime}}(U)=\mathcal{O}\left(U, X^{\prime}\right) /\left(z-T^{\prime}\right) \mathcal{O}\left(U, X^{\prime}\right)
$$

can be identified with the strong dual of the complete nuclear $(D F)$-space

$$
G_{T}(U)=\operatorname{Ker}\left(\mathcal{O}(U)^{\prime} \hat{\otimes} X \stackrel{z-T}{\longrightarrow} \mathcal{O}(U)^{\prime} \hat{\otimes} X\right) .
$$

Suppose that $T \in L(X)$ and $S \in L(Y)$ are quasisimilar operators on Banach spaces $X$ and $Y$. Then, for each open subset $U$ of $\rho_{\mathrm{e}}(T)$, there are topological isomorphisms of nuclear Fréchet spaces

$$
\mathcal{F}_{T}(U) \cong \mathcal{O}(U, Y) / \overline{(z-S) \mathcal{O}(U, Y)}, \quad \mathcal{F}_{T^{\prime}}(U) \cong \mathcal{O}\left(U, Y^{\prime}\right) /{\overline{\left(z-S^{\prime}\right) \mathcal{O}\left(U, Y^{\prime}\right)}}^{w^{*}}
$$

The above identifications hold without taking closures on the right-hand side if $(\partial U) \cap$ $\sigma_{\mathrm{e}}(S)=\emptyset$ and if $S$ satisfies the single-valued extension property near $\partial U$. In this case the nuclearity of the spaces on the right implies that $U \cap \sigma_{\mathrm{e}}(S)=\emptyset$. These observations allow us to prove results on the equality of the essential spectra of suitable quasisimilar operators, and they can be used to give straightforward proofs of the cited results of Herrero.

In $\S 3$ we study the properties of the multiplication operator $\mathcal{O}(U, X) \stackrel{z-T}{\longrightarrow} \mathcal{O}(U, X)$ on the open subsets $U$ of the essential resolvent set $\rho_{\mathrm{e}}(T)$ of $T$, and we use the coherence of the associated kernel and image sheaves to prove results on the equality of essential spectra of quasisimilar operators. Thus, we obtain extensions of results of Putinar [12], Yang [14] and Miller and Miller [11]. In $\S 4$ we use the same coherence principle to give new proofs of the results of Herrero explained above.

\section{Preliminaries}

Let $T \in L(X)$ be a continuous linear operator on a complex Banach space $X$. For each open set $U$ in $\mathbb{C}$, we denote by $\mathcal{O}(U, X)$ the Fréchet space of all $X$-valued analytic functions on $U$. The operator $T \in L(X)$ is said to possess the single-valued extension property on $U$ if the multiplication operator

$$
\mathcal{O}(V, X) \rightarrow \mathcal{O}(V, X), \quad f \mapsto(z-T) f
$$

is injective for each open subset $V$ of $U$. If the same map is injective with closed range for each open subset $V$ of $U$, then $T$ is said to possess Bishop's property $(\beta)$ on $U$. 
The operator $T \in L(X)$ satisfies property $(\delta)$ on $U$ if, for each open subset $V$ of $U$, the operator

$$
\mathcal{O}(V)^{\prime} \hat{\otimes} X \rightarrow \mathcal{O}(V)^{\prime} \hat{\otimes} X, \quad u \mapsto(z-T) u
$$

is surjective or, equivalently, if

$$
X=X_{T}(\bar{W})+X_{T}(\mathbb{C} \backslash V)
$$

holds for each pair of open sets $V, W$ in $\mathbb{C}$ with $\mathbb{C} \backslash U \subset V \subset \bar{V} \subset W$. Here, $\mathcal{O}(V)^{\prime}$ is the strong dual of the Fréchet space $\mathcal{O}(V)$, and, for a given closed set $F$ in $\mathbb{C}$,

$$
X_{T}(F)=\{x \in X ; x \in(z-T) \mathcal{O}(\mathbb{C} \backslash F, X)\}
$$

is the spectral subspace of $T$ with respect to $F$. For details of the above notions from local spectral theory (in particular, the claimed equivalence), the reader is referred to [1].

The operator $T \in L(X)$ is decomposable on $U$ (or modulo $\mathbb{C} \backslash U$ ) if $T$ satisfies property $(\beta)$ and property $(\delta)$ on $U$, or, equivalently, if, for each finite open cover $\left(U_{i}\right)_{1 \leqslant i \leqslant n}$ of the complex plane $\mathbb{C}$ with $\mathbb{C} \backslash U \subset U_{1}$, there are closed invariant subspaces $X_{1}, \ldots, X_{n}$ for $T$ such that

$$
X=X_{1}+\cdots+X_{n}, \quad \sigma\left(T \mid X_{i}\right) \subset U_{i} \quad(i=1, \ldots, n) .
$$

Let $T^{\prime} \in L\left(X^{\prime}\right)$ be the adjoint of $T$ on the dual space $X^{\prime}$ of $X$. It was shown in [1] that $T$ satisfies Bishop's property $(\beta)$ on $U$ if and only if $T^{\prime}$ satisfies $(\delta)$ on $U$, and that this equivalence also holds if the roles of property $(\beta)$ and property $(\delta)$ are exchanged. Furthermore, property $(\beta)$ on $U$ characterizes the restrictions of operators that are decomposable on $U$, while property $(\delta)$ on $U$ characterizes the quotients of operators that are decomposable on $U$.

We regard the space $\mathcal{O}\left(U, X^{\prime}\right)=\mathcal{O}(U) \hat{\otimes} X^{\prime}$ as the strong dual of the Fréchet space $\mathcal{O}(U)^{\prime} \hat{\otimes} X$. In general, we write $\hat{\otimes}$ for the completed $\pi$-tensor product. Since $\mathcal{O}(U)$ and $\mathcal{O}(U)^{\prime}$ are nuclear, the $\pi$ - and $\varepsilon$-tensor products coincide in the above cases. We speak of the $w^{*}$-topology of $\mathcal{O}\left(U, X^{\prime}\right)$, when we mean the weak topology induced on $\mathcal{O}\left(U, X^{\prime}\right)$ by the duality $\left\langle\mathcal{O}(U)^{\prime} \hat{\otimes} X, \mathcal{O}\left(U, X^{\prime}\right)\right\rangle$. Suppose that the operator $T \in L(X)$ satisfies Bishop's property $(\beta)$ on a given open set $U$ in $\mathbb{C}$. Then the analytic Fréchet sheaf on $U$, given by the pre-sheaf of Fréchet spaces

$$
\mathcal{F}_{T}(V)=\mathcal{O}(V, X) /(z-T) \mathcal{O}(V, X) \quad(V \subset U \text { open })
$$

together with the canonical restriction maps, is called the sheaf model of $T$ on $U$.

Let $E$ be a Fréchet space. The space $E^{\infty}$ consisting of all bounded sequences in $E$ is a Fréchet space relative to the seminorms

$$
p_{\infty}(x)=\sup _{n} p\left(x_{n}\right) \quad\left(x=\left(x_{n}\right)_{n \geqslant 0} \in E^{\infty}\right)
$$

where $p$ runs through all continuous seminorms on $E$. The space $E^{\text {pc }}$ of all sequences $\left(x_{n}\right)$ in $E$ such that each subsequence of $\left(x_{n}\right)$ has a convergent subsequence is a closed subspace of $E^{\infty}$. Hence, the quotient $E^{q}=E^{\infty} / E^{\mathrm{pc}}$ is a Fréchet space with respect to 
the quotient topology. Each continuous linear operator $T \in L(E)$ induces continuous linear operators $T^{\infty}: E^{\infty} \rightarrow E^{\infty},\left(x_{n}\right) \mapsto\left(T x_{n}\right), T^{\mathrm{pc}}=T^{\infty} \mid E^{\mathrm{pc}}$, and $T^{q}=T^{\infty} / E^{\mathrm{pc}}$. Let $U \subset \mathbb{C}$ be open, and let $X$ be a Banach space. There is a canonical topological isomorphism $\mathcal{O}(U, X)^{q} \cong \mathcal{O}\left(U, X^{q}\right)$ of Fréchet spaces.

The functor assigning to each Fréchet space $E$ the Fréchet space $E^{q}$ is left exact in the sense that, for each short exact sequence

$$
0 \rightarrow E \stackrel{\alpha}{\longrightarrow} F \stackrel{\beta}{\rightarrow} G \rightarrow 0
$$

of Fréchet spaces, the induced sequence

$$
0 \rightarrow E^{q} \stackrel{\alpha^{q}}{\longrightarrow} F^{q} \stackrel{\beta^{q}}{\longrightarrow} G^{q}
$$

is exact. Since a continuous surjection between Fréchet spaces allows the lifting of precompact sequences (see [8]), the map $\alpha^{q}: E^{q} \rightarrow F^{q}$ is a topological isomorphism whenever $G^{q}=0$, i.e. $G$ is an $(F M)$-space.

For $T \in L(X)$, we denote by $\sigma(T)$ the spectrum of $T$ and by

$$
\sigma_{\mathrm{e}}(T)=\{\lambda \in \mathbb{C} ; \operatorname{dim} \operatorname{Ker}(\lambda-T)=\infty \text { or } \operatorname{dim} X / \operatorname{Im}(\lambda-T)=\infty\},
$$

the essential spectrum of $T$. We write $\rho(T)=\mathbb{C} \backslash \sigma(T)$ and $\rho_{\mathrm{e}}(T)=\mathbb{C} \backslash \sigma_{\mathrm{e}}(T)$ for the resolvent set and the essential resolvent set of $T$, respectively. It is well known (cf. $\S 2.6$ in [3]) that

$$
\sigma\left(T^{q}\right)=\sigma_{\mathbf{e}}(T)
$$

Finally, let us recall that two operators $T \in L(X)$ and $S \in L(Y)$ on Banach spaces $X$ and $Y$ are quasisimilar if there are injective continuous linear operators $A: X \rightarrow Y$ and $B: Y \rightarrow X$ with dense range such that $S A=A T$ and $T B=B S$.

\section{Coherent analytic sheaves and Fredholm theory}

Let $T \in L(X)$ be a continuous linear operator on a complex Banach space $X$. The proofs of our results on the Fredholm structure of Banach space operators will be based on the following observation concerning the behaviour of $T$ on its Fredholm domain.

Theorem 3.1. Let $T \in L(X)$ be a continuous linear operator on a complex Banach space $X$. For each open set $U \subset \rho_{\mathrm{e}}(T)$, the map

$$
\mathcal{O}(U, X) \stackrel{z-T}{\longrightarrow} \mathcal{O}(U, X)
$$

has closed range, and the map

$$
\mathcal{O}\left(U, X^{\prime}\right) \stackrel{z-T^{\prime}}{\longrightarrow} \mathcal{O}\left(U, X^{\prime}\right)
$$

has $w^{*}$-closed range.

The proof depends on a standard construction from the theory of analytically parametrized Fredholm complexes (see Proposition 9.4.5 in [3]), which will be explained in the following lemmas. 
Lemma 3.2. For each point $z_{0} \in \rho_{\mathrm{e}}(T)$, there is an open neighbourhood $V$ of $z_{0}$ such that the map

$$
\mathcal{O}(W, X) \stackrel{z-T}{\longrightarrow} \mathcal{O}(W, X)
$$

has closed range for each open subset $W$ of $V$.

Proof. Fix $z_{0} \in \rho_{\mathrm{e}}(T)$ and a basis $\left\{\left[y_{1}\right], \ldots,\left[y_{r}\right]\right\}$ of $X /\left(z_{0}-T\right) X$. Define $L^{1}=\mathbb{C}^{r}$ and

$$
h^{1}: L^{1} \rightarrow X, \quad\left(t_{i}\right)_{i=1}^{r} \mapsto \sum_{i=1}^{r} t_{i} y_{i}
$$

If $z_{0}-T$ is onto, then we set $L^{1}=\{0\}$ and $h^{1}=0$. On a suitable open neighbourhood $V$ of $z_{0}$, the induced map

$$
\mathcal{O}_{V}^{X} \oplus \mathcal{O}_{V}^{L^{1}} \stackrel{\left(z-T,-h^{1}\right)}{\longrightarrow} \mathcal{O}_{V}^{X}
$$

is onto (Lemma 2.1.5 in [3]). Fix a basis $\left\{x_{1}, \ldots, x_{s}\right\}$ of $\operatorname{Ker}\left(z_{0}-T\right.$ ). After shrinking $V$, we may suppose (see the remark following Lemma 2.1 .5 in [3]) that there are analytic functions $f_{1}, \ldots, f_{s} \in \mathcal{O}\left(V, X \oplus L^{1}\right)$ with $f_{i}\left(z_{0}\right)=x_{i}(1 \leqslant i \leqslant s)$ and

$$
\left(z-T,-h_{1}\right) f_{i}(z)=0 \quad(z \in V, 1 \leqslant i \leqslant s) .
$$

Define $L^{0}=\mathbb{C}^{s}\left(=\{0\}\right.$ if $z_{0}-T$ is injective). The operators

$$
\begin{array}{ll}
d^{1}(z): X \oplus L^{1} \rightarrow X, \quad(x, y) \mapsto(z-T) x-h^{1} y, \\
d^{0}(z): L^{0} \rightarrow X \oplus L^{1}, \quad\left(t_{i}\right)_{i=1}^{s} \mapsto \sum_{i=1}^{s} t_{i} f_{i}(z),
\end{array}
$$

depend analytically on $z \in V$. After shrinking $V$ again, if necessary, we may suppose that the complexes

$$
0 \rightarrow L^{0} \stackrel{d^{0}(z)}{\longrightarrow} X \oplus L^{1} \stackrel{d^{1}(z)}{\longrightarrow} X \rightarrow 0 \quad(z \in V),
$$

as well as the induced complex

$$
0 \rightarrow \mathcal{O}_{V}^{L^{0}} \stackrel{d^{0}}{\rightarrow} \mathcal{O}_{V}^{X \oplus L^{1}} \stackrel{d^{1}}{\rightarrow} \mathcal{O}_{V}^{X} \rightarrow 0
$$

are exact (see Theorem 2.1.8 in [3]). Let us write $d^{0}$ in the form $d^{0}=\left(h^{0}, u^{0}\right)^{t}$, where $h^{0} \in \mathcal{O}\left(V, L\left(L^{0}, X\right)\right)$ and $u^{0} \in \mathcal{O}\left(V, L\left(L^{0}, L^{1}\right)\right)$. Then the last short exact sequence can be regarded as the mapping cone (see Appendix 2 in [3]) of the morphism of complexes

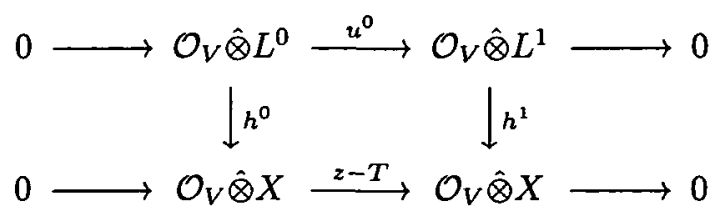

given by $h^{0}$ and $h^{1}$. 
After shrinking $V$ one more time, we may suppose that there is an analytic operatorvalued function $\varepsilon^{1}=\left(k^{0}, v^{1}\right): V \rightarrow L\left(X \oplus L^{1}, L^{0}\right)$ with $k^{0} \in \mathcal{O}\left(V, L\left(X, L^{0}\right)\right)$ and $v^{1} \in \mathcal{O}\left(V, L\left(L^{1}, L^{0}\right)\right)$ such that

$$
\varepsilon^{1}(z) \circ d^{0}(z)=I_{L^{0}} \quad(z \in V) .
$$

To see this, note that, for each $z \in V$, the map

$$
R(z): L\left(X \oplus L^{1}, L^{0}\right) \rightarrow L\left(L^{0}\right), \quad T \mapsto T d^{0}(z)
$$

is onto, and then apply Lemma 2.1 .5 from [3]. The map

$$
P: V \rightarrow L\left(X \oplus L^{1}\right), \quad P(z)=d^{0}(z) \varepsilon^{1}(z)
$$

is an analytic projection-valued function with $\operatorname{Im} P(z)=\operatorname{Im} d^{0}(z)=\operatorname{Ker} d^{1}(z)(z \in V)$. For $z \in V$, define the operator $\varepsilon^{2}(z)=\left(r^{1}(z),-k^{1}(z)\right)^{t} \in L\left(X, X \oplus L^{1}\right)$ as the inverse of

$$
(I-P(z))\left(X \oplus L^{1}\right) \stackrel{d^{1}(z)}{\sim} X,
$$

regarded as a map with values in $X \oplus L^{\mathbf{1}}$. After shrinking $V$ again, we may suppose that $\varepsilon^{2}: V \rightarrow L\left(X, X \oplus L^{1}\right)$ is analytic. Indeed, for $z$ close enough to $z_{0}$, the operators $\tilde{d}^{1}(z)$ acting as

$$
\left(I-P\left(z_{0}\right)\right)\left(X \oplus L^{1}\right) \stackrel{d^{1}(z)}{\longrightarrow} X
$$

are invertible, and

$$
d^{1}(z)(I-P(z)) \tilde{d}^{1}(z)^{-1}=I_{X} .
$$

Hence, $\varepsilon^{2}(z)=(I-P(z)) \tilde{d}^{1}(z)^{-1}$ is analytic for $z$ close enough to $z_{0}$.

The functions $\varepsilon^{1}$ and $\varepsilon^{2}$ form a splitting for the mapping cone of the morphism $h=$ $\left(h^{i}\right)_{i=0,1}$. Indeed, our definitions imply that, for $z \in V$,

(i) $\varepsilon^{1}(z) \varepsilon^{2}(z)=0$;

(ii) $\varepsilon^{1}(z) d^{0}(z)=I_{L^{0}}, d^{1}(z) \varepsilon^{2}(z)=I_{X}$; and

(iii) $d^{0}(z) \varepsilon^{1}(z)+\varepsilon^{2}(z) d^{1}(z)=I_{X \oplus L^{1}}$.

Hence, $h=\left(h^{i}\right)_{i=0,1}$ is a homotopy equivalence with homotopy inverse given by $k=$ $\left(k^{i}\right)_{i=0,1}$. Indeed,

$$
k^{0}(z) h^{0}(z)=I_{L^{0}}-v^{1}(z) u^{0}(z), \quad k^{1}(z) h^{1}(z)=I_{L^{1}}-u^{0}(z) v^{1}(z)
$$

and

$$
h^{0}(z) k^{0}(z)=I_{X}-r^{1}(z)(z-T), \quad h^{1}(z) k^{1}(z)=I_{X}-(z-T) r^{1}(z)
$$

holds for $z \in V$. 
In particular, for each open set $W \subset V$, the map

$$
\mathcal{O}(W) \hat{\otimes} L^{1} / u^{0} \mathcal{O}(W) \hat{\otimes} L^{0} \stackrel{h^{1}}{\rightarrow} \mathcal{O}(W, X) /(z-T) \mathcal{O}(W, X)
$$

induced by $h^{1}$, is a vector-space isomorphism. Its inverse is the corresponding map induced by $k^{1}$. Both maps are continuous with respect to the quotient topologies, and the space on the left is a Fréchet space in its quotient topology (see the 'Abgeschlossenheitssatz' in [5, p. 172]). Hence, for each open set $W \subset V$, the map

$$
\mathcal{O}(W, X) \stackrel{z-T}{\longrightarrow} \mathcal{O}(W, X)
$$

has closed range.

To prove the second part of Theorem 3.1 we need the dual version of Lemma 3.2.

Lemma 3.3. For each point $z_{0} \in \rho_{\mathrm{e}}(T)$, there is an open neighbourhood $V$ of $z_{0}$ such that the map

$$
\mathcal{O}\left(W, X^{\prime}\right) \stackrel{z-T^{\prime}}{\longrightarrow} \mathcal{O}\left(W, X^{\prime}\right)
$$

has $w^{*}$-closed range for each open subset $W$ of $V$.

Proof. Let us indicate what happens if everywhere in the last proof the space $X$ is replaced by its dual $X^{\prime}$ and the operator $T \in L(X)$ is replaced by its adjoint $T^{\prime} \in$ $L\left(X^{\prime}\right)$. Note that $L^{0} \cong\left(L^{0 \prime}\right)^{\prime}$ and $L^{1} \cong\left(L^{1 \prime}\right)^{\prime}$ and that the $w^{*}$-topologies of $L^{0}$ and $L^{1}$ with respect to these dualities coincide with the norm-topologies. The operators $\varepsilon^{1}(z) \in$ $L\left(X^{\prime} \oplus L^{1}, L^{0}\right)$ can be chosen as $w^{*}$-continuous linear operators. Indeed, since each finitedimensional subspace of $X^{\prime} \oplus L^{1}$ has a $w^{*}$-closed direct complement in $X^{\prime} \oplus L^{1}$, also in this case the operators

$$
R(z): \mathcal{L}\left(X^{\prime} \oplus L^{1}, L^{0}\right) \rightarrow L\left(L^{0}\right), \quad T \mapsto T d^{0}(z),
$$

where $\mathcal{L}\left(X^{\prime} \oplus L^{1}, L^{0}\right)$ is the closed subspace of $L\left(X^{\prime} \oplus L^{1}, L^{0}\right)$ consisting of all $w^{*}$ continuous operators, are surjective.

However, the operators $P(z)=d^{0}(z) \varepsilon^{1}(z) \in L\left(X^{\prime} \oplus L^{1}\right), \varepsilon^{2}(z) \in L\left(X^{\prime}, X^{\prime} \oplus L^{1}\right)$ and $k^{1}(z) \in L\left(X^{\prime}, L^{1}\right)$ are then also $w^{*}$-continuous for each $z \in V$. As before, for each open set $W \subset V$, the operator

$$
\mathcal{O}\left(W, X^{\prime}\right) /\left(z-T^{\prime}\right) \mathcal{O}\left(W, X^{\prime}\right) \stackrel{k^{1}}{\rightarrow} \mathcal{O}\left(W, L^{1}\right) / u^{0} \mathcal{O}\left(W, L^{0}\right)
$$

is a vector-space isomorphism. Since $\mathcal{O}\left(W, L^{1}\right)$ is a reflexive space, the proof of Lemma 3.3 is complete, if we can show that the operator

$$
\mathcal{O}\left(W, X^{\prime}\right) \stackrel{k^{1}}{\rightarrow} \mathcal{O}\left(W, L^{1}\right)
$$

is $w^{*}$-continuous. We regard $\mathcal{O}\left(W, X^{\prime}\right)$ and $\mathcal{O}\left(W, L^{1}\right)$ here as the dual spaces of $\mathcal{O}(W)^{\prime} \hat{\otimes} X$ and $\mathcal{O}(W)^{\prime} \hat{\otimes}\left(L^{1}\right)^{\prime}$. Thus, the next result completes the proof. 
Proposition 3.4. Let $E, F$ be complex Banach spaces, and let $\mathcal{L}\left(F^{\prime}, E^{\prime}\right)$ be the closed subspace of $L\left(F^{\prime}, E^{\prime}\right)$ consisting of all $w^{*}$-continuous linear operators. For each open set $W$ in $\mathbb{C}$, the map

$$
\chi: \mathcal{O}(W) \times \mathcal{L}\left(F^{\prime}, E^{\prime}\right) \rightarrow L_{b}\left(\mathcal{O}(W)^{\prime} \hat{\otimes} E, \mathcal{O}(W)^{\prime} \hat{\otimes} F\right), \quad\left(f, T^{\prime}\right) \mapsto\left(M_{f}^{\prime}\right) \otimes T
$$

is continuous bilinear and induces a continuous linear map

$$
\hat{\chi}: \mathcal{O}\left(W, \mathcal{L}\left(F^{\prime}, E^{\prime}\right)\right) \rightarrow L_{b}\left(\mathcal{O}(W)^{\prime} \hat{\otimes} E, \mathcal{O}(W)^{\prime} \hat{\otimes} F\right) .
$$

For $h \in \mathcal{O}\left(W, \mathcal{L}\left(F^{\prime}, E^{\prime}\right)\right)$, the adjoint of the operator $\hat{\chi}(h)$ is the multiplication operator

$$
\mathcal{O}\left(W, F^{\prime}\right) \stackrel{h}{\rightarrow} \mathcal{O}\left(W, E^{\prime}\right)
$$

Proof. To prove the continuity of $\chi$, it is sufficient to prove its separate continuity (see $[9, \S 40.2 .(1)])$. One can prove the separate continuity of $\chi$ directly by using the fact that each bounded set in $\mathcal{O}(W)^{\prime} \hat{\otimes} E$ is contained in a set of the form (see [3, Corollary A.1.11]) $\overline{\Gamma(C \times D)}$, where $C$ and $D$ are bounded sets in $\mathcal{O}(W)^{\prime}$ and $E$ ( $\Gamma$ stands for the absolutely convex hull), and by using the fact that the topology of $\mathcal{O}(W)^{\prime} \hat{\otimes} F$ is generated by the continuous extensions of all seminorms $p$ on $\mathcal{O}(W)^{\prime} \otimes F$ of the form (see $[9, \S 41.2]$ )

$$
p(z)=\inf \left\{\sum_{i=1}^{n}\left\|u_{i}\right\|_{M}\left\|x_{i}\right\|_{;} z=\sum_{i=1}^{n} u_{i} \otimes x_{i}\right\},
$$

where $M$ runs through all bounded sets in $\mathcal{O}(W)$ and

$$
\|u\|_{M}=\sup _{f \in M}|u(f)|
$$

Since $\mathcal{O}(W)^{\prime} \hat{\otimes} E$ is a $(D F)$-space (see $[9, \S 41.4(7)]$ ), the space

$$
L_{b}\left(\mathcal{O}(W)^{\prime} \hat{\otimes} E, \mathcal{O}(W)^{\prime} \hat{\otimes} F\right)
$$

is complete (see $[8, \S 29.3(7)]$ ), and $\chi$ induces a continuous linear map $\hat{\chi}$, as in the assertion. To prove the last part of Proposition 3.4, it suffices to observe that

$$
\left\langle\hat{\chi}\left(f \otimes T^{\prime}\right)(u \otimes x), g \otimes v\right\rangle=\left\langle u \otimes x,(f g) \otimes\left(T^{\prime} v\right)\right\rangle
$$

for all $f, g \in \mathcal{O}(W), x \in E, T \in L(E, F)$, and $u \in \mathcal{O}(W)^{\prime}, v \in F^{\prime}$.

Up to now we have proved that the assertion of Theorem 3.1 holds locally. To obtain the global version of the first part of Theorem 3.1 we use some elementary arguments from the theory of coherent sheaves.

It is well known, and it follows from the proof of Lemma 3.2, that the cohomology sheaves,

$$
\mathcal{H}^{0}\left(z-T, \mathcal{O}_{\mathbb{C}}^{X}\right)=\operatorname{Ker}\left(\mathcal{O}_{\mathbb{C}}^{X} \stackrel{z-T}{\longrightarrow} \mathcal{O}_{\mathbb{C}}^{X}\right)
$$




$$
\mathcal{H}^{1}\left(z-T, \mathcal{O}_{\Im}^{X}\right)=\mathcal{O}_{\widetilde{c}}^{X} /(z-T) \mathcal{O}_{\cong}^{X}
$$

restricted to $\rho_{\mathrm{e}}(T)$ are coherent analytic sheaves (see [3, Proposition 10.1.3]).

Since all sheaves in the exact sequences

$$
\begin{gathered}
0 \rightarrow \mathcal{H}^{0}\left(z-T, \mathcal{O}_{\mathbb{C}}^{X}\right) \stackrel{i}{\rightarrow} \mathcal{O}_{\mathbb{\complement}}^{X} \stackrel{z-T}{\rightarrow}(z-T) \mathcal{O}_{\mathbb{C}}^{X} \rightarrow 0 \\
0 \rightarrow(z-T) \mathcal{O}_{\mathbb{C}}^{X} \stackrel{i}{\rightarrow} \mathcal{O}_{\mathbb{C}}^{X} \stackrel{q}{\rightarrow} \mathcal{O}_{\mathbb{C}}^{X} /(z-T) \mathcal{O}_{\mathbb{C}}^{X} \rightarrow 0
\end{gathered}
$$

are acyclic on each open subset $U$ of $\rho_{\mathrm{e}}(T)$ (cf. [10, Satz 18.4]), there are canonical vector-space isomorphisms

$$
\mathcal{O}(U, X) /(z-T) \mathcal{O}(U, X) \stackrel{\sim}{\longrightarrow} \Gamma\left(U, \mathcal{O}_{\mathbb{C}}^{X} /(z-T) \mathcal{O}_{\mathbb{C}}^{X}\right) \quad\left(U \subset \rho_{\mathrm{e}}(T) \text { open }\right) .
$$

Let us equip the spaces on the left with their quotient topologies, and let us equip the spaces on the right with their canonical nuclear Fréchet space topologies (see [5, ch. V, §6]). Since homomorphisms between coherent sheaves induce continuous linear maps between section spaces, the proof of Lemma 3.2 shows that each point $z_{0} \in \rho_{\mathrm{e}}(T)$ possesses an open neighbourhood $V$ such that the above vector-space isomorphisms are topological isomorphisms for all open subsets $U$ of $V$.

For a given open set $U \subset \rho_{\mathrm{e}}(T)$, we choose an open cover $\left(U_{i}\right)$ of $U$ by countably many open sets $U_{i}$, such that the components of the right vertical map in the commutative diagram

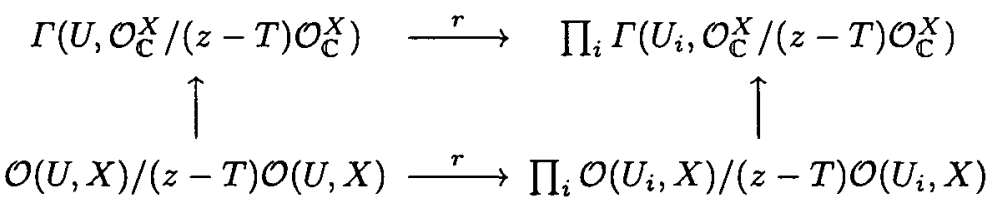

are topological isomorphisms. Since the upper horizontal map is a topological monomorphism, the left vertical map is continuous. It follows that $(z-T) \mathcal{O}(U, X)$ is closed, and that the left vertical map is even a topological isomorphism between nuclear Fréchet spaces.

Thus, we have proved the first part of Theorem 3.1. To prove the global version of the second part of Theorem 3.1, we use duality theory for locally convex spaces. Let $T \in L(X)$ be a continuous linear operator on a complex Banach space. For $U \subset \mathbb{C}$ open, we define

$$
\begin{aligned}
E(U) & =\mathcal{O}\left(U, X^{\prime}\right) /\left(z-T^{\prime}\right) \mathcal{O}\left(U, X^{\prime}\right) \\
M(U) & =\operatorname{Ker}\left(\mathcal{O}(U)^{\prime} \hat{\otimes} X \stackrel{z-T}{\longrightarrow} \mathcal{O}(U)^{\prime} \hat{\otimes} X\right)
\end{aligned}
$$

We equip the first space with its quotient topology, and we regard the second space as a closed subspace of the $(D F)$-space $\mathcal{O}(U)^{\prime} \hat{\otimes} X$. The missing part of Theorem 3.1 is proved as a part of the next result. 
Theorem 3.5. For each open set $U \subset \rho_{\mathrm{e}}(T)$, the range of the operator

$$
\mathcal{O}\left(U, X^{\prime}\right) \stackrel{z-T^{\prime}}{\longrightarrow} \mathcal{O}\left(U, X^{\prime}\right)
$$

is $w^{*}$-closed. With respect to the duality $\left\langle\mathcal{O}(U)^{\prime} \hat{\otimes} X, \mathcal{O}(U) \hat{\otimes} X^{\prime}\right\rangle$, the nuclear Fréchet space

$$
E(U)=\mathcal{O}\left(U, X^{\prime}\right) /\left(z-T^{\prime}\right) \mathcal{O}\left(U, X^{\prime}\right)
$$

is the strong dual of the complete nuclear $(D F)$-space

$$
M(U)=\operatorname{Ker}\left(\mathcal{O}(U)^{\prime} \hat{\otimes} X \stackrel{z-T}{\longrightarrow} \mathcal{O}(U)^{\prime} \hat{\otimes} X\right) .
$$

Proof. Let us fix an open set $U \subset \rho_{\mathrm{e}}(T)$. Let us suppose that we know already that $\left(z-T^{\prime}\right) \mathcal{O}\left(U, X^{\prime}\right)$ is a $w^{*}$-closed subspace of $\mathcal{O}\left(U, X^{\prime}\right)$.

Since the quotient space $E=E(U)$ is a nuclear Fréchet space (by the proof of the first part of Theorem 3.1), its strong dual space $E_{b}^{\prime}$ can be identified topologically with the closed subspace

$$
\operatorname{Ker}\left(\mathcal{O}(U)^{\prime} \hat{\otimes} X^{\prime \prime} \stackrel{z-T^{\prime \prime}}{\longrightarrow} \mathcal{O}(U)^{\prime} \hat{\otimes} X^{\prime \prime}\right)
$$

of $\mathcal{O}(U)^{\prime} \hat{\otimes} X^{\prime \prime}$ (see $[8, \S 22.2(7)]$ and $[9, \S 32.5(2)]$ ). Hence, the space $M=M(U)$ can be regarded as a closed subspace of the strong dual $E_{b}^{\prime}$ of the nuclear Fréchet space $E$. Since $E$ is reflexive, $M$ is also $\sigma\left(E^{\prime}, E\right)$-closed as a subspace of $E_{b}^{\prime}$.

Since the quotient space $E /^{\perp} M$ is nuclear, a repetition of the above argument shows that the strong dual topology on $M$ induced by the duality $\left\langle E /^{\perp} M, M\right\rangle$ coincides with the relative topology of $M$ as a subspace of $E_{b}^{\prime}$. But then the subspace $M \subset \mathcal{O}(U)^{\prime} \hat{\otimes} X$ is a complete nuclear $(D F)$-space. In particular, the strong dual topology induced by $M$ on $\mathcal{O}(U) \hat{\otimes} X^{\prime} / M^{\perp}=E$ is the quotient topology of the natural Fréchet-space topology on $\mathcal{O}(U)^{\prime} \hat{\otimes} X$ (see $\left.[8, \S 29.5(2)]\right)$. As the strong dual of a nuclear Fréchet space, the space $M$ is reflexive.

Let $U \subset \rho_{\mathbf{e}}(T)$ be an arbitrary open set. Choose a countable open cover $\left(U_{i}\right)$ of $U$ such that $\left(z-T^{\prime}\right) \mathcal{O}\left(U_{i}, X^{\prime}\right) \subset \mathcal{O}\left(U_{i}, X^{\prime}\right)$ is $w^{*}$-closed for each $i$. Consider the commutative diagram that was used in the proof of the first part of Theorem 3.1, but this time with $X$ replaced by $X^{\prime}$ and $T$ replaced by $T^{\prime}$. The maps

$$
\mathcal{O}\left(U_{i}, X^{\prime}\right) /\left(z-T^{\prime}\right) \mathcal{O}\left(U_{i}, X^{\prime}\right) \rightarrow \Gamma\left(U_{i}, \mathcal{O}_{\mathbb{C}}^{X^{\prime}} /\left(z-T^{\prime}\right) \mathcal{O}_{\mathbb{C}}^{X^{\prime}}\right)
$$

are continuous if the spaces on the left are equipped with the quotient topology of the weak*-topology of $\mathcal{O}\left(U_{i}, X^{\prime}\right)$, and if the spaces on the right are equipped with their weak topology (see [13, Corollary 1, Chapter IV.4.1]).

Since the topological monomorphism

$$
\Gamma\left(U, \mathcal{O}_{\mathbb{C}}^{X^{\prime}} /\left(z-T^{\prime}\right) \mathcal{O}_{\mathbb{C}}^{X^{\prime}}\right) \rightarrow \prod_{i} \Gamma\left(U_{i}, \mathcal{O}_{\mathbb{C}}^{X^{\prime}} /\left(z-T^{\prime}\right) \mathcal{O}_{\mathbb{C}}^{X^{\prime}}\right)
$$

is also a weak monomorphism (see $[9, \S 32.3(3)]$ ), it follows that the quotient space $\mathcal{O}\left(U, X^{\prime}\right) /\left(z-T^{\prime}\right) \mathcal{O}\left(U, X^{\prime}\right)$ equipped with the quotient topology of the weak ${ }^{*}$-topology of $\mathcal{O}\left(U, X^{\prime}\right)$ is Hausdorff. Hence, $\left(z-T^{\prime}\right) \mathcal{O}\left(U, X^{\prime}\right) \subset \mathcal{O}\left(U, X^{\prime}\right)$ is $w^{*}$-closed. 
Since the space $M(U)$ is reflexive, we can also regard the space $M(U)$ as the strong dual space of the nuclear Fréchet space $E(U)$.

As a first application we prove natural extensions of results of Putinar [12] and Yang [14] on the equality of the essential spectra of quasisimilar operators with Bishop's property $(\beta)$.

Corollary 3.6. Let $T \in L(X)$ and $S \in L(Y)$ be continuous linear operators on complex Banach spaces $X$ and $Y$. Let $A: X \rightarrow Y$ and $B: Y \rightarrow X$ be continuous linear operators with $A T=S A$ and $B S=T B$.

(a) If $A$ and $B$ have dense range, then

$$
\mathcal{O}(U, X) /(z-T) \mathcal{O}(U, X) \stackrel{A}{\rightarrow} \mathcal{O}(U, Y) / \overline{(z-S) \mathcal{O}(U, Y)}
$$

is a topological isomorphism of nuclear Fréchet spaces for each open set $U \subset \rho_{\mathrm{e}}(T)$.

(b) If $A$ and $B$ are injective, then

$$
\mathcal{O}\left(U, X^{\prime}\right) /\left(z-T^{\prime}\right) \mathcal{O}\left(U, X^{\prime}\right) \stackrel{B^{\prime}}{\longrightarrow} \mathcal{O}\left(U, Y^{\prime}\right) /{\overline{\left(z-S^{\prime}\right) \mathcal{O}\left(U, Y^{\prime}\right)}}^{w^{*}}
$$

is a topological isomorphism of nuclear Fréchet spaces for each open set $U \subset \rho_{\mathrm{e}}(T)$.

Proof. (a) The quotient sheaf $\mathcal{F}=\mathcal{O}_{\rho_{\mathrm{e}}(T)}^{X} /(z-T) \mathcal{O}_{\rho_{\mathrm{e}}(T)}^{X}$ is a coherent sheaf with section spaces $\mathcal{F}(U)=\mathcal{O}(U, X) /(z-T) \mathcal{O}(U, X)\left(U \subset \rho_{\mathrm{e}}(T)\right.$ open). Suppose that $A$ and $B$ have dense range. Then the induced maps $B A: \mathcal{F}(U) \rightarrow \mathcal{F}(U)$ have dense range, and they yield a sheaf homomorphism $B A: \mathcal{F} \rightarrow \mathcal{F}$. Since the image sheaf $(B A) \mathcal{F} \subset \mathcal{F}$ is a coherent subsheaf, the space

$$
(B A) \mathcal{F}(U)=((B A) \mathcal{F})(U) \subset \mathcal{F}(U)
$$

is a closed subspace of the Fréchet space $\mathcal{F}(U)$ for each open set $U \subset \rho_{\mathrm{e}}(T)$ (Abgeschlossenheitssatz in [5, p. 172]). Hence, the sheaf homomorphism $B A: \mathcal{F} \rightarrow \mathcal{F}$ is onto. Since, for each $z \in \rho_{\mathrm{e}}(T)$, the sequence of submodules $\operatorname{Ker}(B A)_{z}^{k}(k \geqslant 1)$ of the noetherian $\mathcal{O}_{z}$-module $\mathcal{F}_{z}$ becomes stable, the sheaf homomorphism $B A: \mathcal{F} \rightarrow \mathcal{F}$ is also injective.

Let $U \subset \rho_{\mathrm{e}}(T)$ be open. Since the topological isomorphism $B A: \mathcal{F}(U) \rightarrow \mathcal{F}(U)$ admits the factorization

$$
\mathcal{O}(U, X) /(z-T) \mathcal{O}(U, X) \stackrel{A}{\rightarrow} \mathcal{O}(U, Y) \overline{(z-S) \mathcal{O}(U, Y)} \stackrel{B}{\rightarrow} \mathcal{O}(U, X) /(z-T) \mathcal{O}(U, X),
$$

the first map is a topological isomorphism.

(b) As in part (a), the quotient sheaf $\mathcal{F}^{\prime}=\mathcal{O}_{\rho_{\mathrm{e}}(T)}^{X^{\prime}} /\left(z-T^{\prime}\right) \mathcal{O}_{\rho_{\mathrm{e}}(T)}^{X^{\prime}}$ is a coherent analytic sheaf with section spaces

$$
\mathcal{F}^{\prime}(U)=\mathcal{O}\left(U, X^{\prime}\right) /\left(z-T^{\prime}\right) \mathcal{O}\left(U, X^{\prime}\right) \quad\left(U \subset \rho_{\mathrm{e}}(T) \text { open }\right) .
$$

Suppose that $A$ and $B$ are injective. Then the maps

$$
A^{\prime} B^{\prime}: \mathcal{O}\left(U, X^{\prime}\right) \rightarrow \mathcal{O}\left(U, X^{\prime}\right) \quad\left(U \subset \rho_{\mathbf{e}}(T) \text { open }\right)
$$


have $w^{*}$-dense range. It follows from Theorem 3.5 that the induced maps $A^{\prime} B^{\prime}: \mathcal{F}^{\prime}(U) \rightarrow$ $\mathcal{F}^{\prime}(U)$ have dense range for each open set $U$ in $\rho_{\mathrm{e}}(T)$. Exactly as in part (a), it follows that the map $A^{\prime} B^{\prime}: \mathcal{F}^{\prime} \rightarrow \mathcal{F}^{\prime}$ is an isomorphism of coherent sheaves, and that the maps

$$
\mathcal{O}\left(U, X^{\prime}\right) /\left(z-T^{\prime}\right) \mathcal{O}\left(U, X^{\prime}\right) \stackrel{B^{\prime}}{\longrightarrow} \mathcal{O}\left(U, Y^{\prime}\right){\overline{\left(z-S^{\prime}\right) \mathcal{O}\left(U, Y^{\prime}\right)}}^{w^{*}} \quad\left(U \subset \rho_{\mathrm{e}}(T) \text { open }\right)
$$

are $w^{*}$-monomorphisms with $w^{*}$-dense range. Equivalently (see $[9, \S 32.3(4)]$ ), the predual maps

$$
\operatorname{Ker}\left(\mathcal{O}(U)^{\prime} \hat{\otimes} Y \stackrel{z-S}{\longrightarrow} \mathcal{O}(U)^{\prime} \hat{\otimes} Y\right) \stackrel{B}{\longrightarrow} \operatorname{Ker}\left(\mathcal{O}(U)^{\prime} \hat{\otimes} X \stackrel{z-T}{\longrightarrow} \mathcal{O}(U)^{\prime} \hat{\otimes} X\right)
$$

are continuous bijections for all open sets $U \subset \rho_{\mathrm{e}}(T)$. As the strong dual of the reflexive Fréchet space $\mathcal{F}^{\prime}(U)$, the space on the right is complete and bornological (see [9, $\S 29.4(4)]$ ), hence ultrabornological. Since the space on the left is webbed (see $[9, \S 35.4]$ ), De Wilde's open-mapping principle implies that the above continuous bijections are topological isomorphisms (see $[9, \S 35.3(1)]$ ). Thus, the proof of part (b) is complete.

By imposing natural extra conditions on the operator $S$, one obtains information on the relation between the spectra and essential spectra of the operators $S$ and $T$.

Corollary 3.7. Let $T \in L(X), S \in L(Y)$ and $A \in L(X, Y), B \in L(Y, X)$ be continuous linear operators on Banach spaces $X$ and $Y$ such that $A T=S A$ and $B S=T B$.

(a) Suppose that $A$ and $B$ have dense range and that $S$ satisfies Bishop's property $(\beta)$ on $\rho_{\mathrm{e}}(T)$. Then $\sigma(S) \subset \sigma(T)$ and $\sigma_{\mathrm{e}}(S) \subset \sigma_{\mathrm{e}}(T)$.

(b) Suppose that $A$ and $B$ are injective and that $S$ satisfies property $(\delta)$ on $\rho_{\mathrm{e}}(T)$. Then $\sigma(S) \subset \sigma(T)$ and $\sigma_{\mathrm{e}}(S) \subset \sigma_{\mathrm{e}}(T)$.

Proof. Let $A, B$ and $S$ be as in part (a), and let $\mathcal{F}_{S}$ be the sheaf model of the operator $S$. By Corollary 3.6, $\mathcal{F}_{S} \mid \rho(T)=0$ and $\mathcal{F}_{S} \mid \rho_{\mathrm{e}}(T)$ is a coherent analytic sheaf. Hence, $\sigma(S) \subset \sigma(T)$ and $\sigma_{\mathrm{e}}(S) \subset \sigma_{\mathrm{e}}(T)$ (see [3, Proposition 10.1.3]).

Secondly, suppose that $A$ and $B$ are injective and that $S$ satisfies property $(\delta)$ on $\rho_{\mathrm{e}}(T)$. It was proved in [2] (or the proof of Theorem 1.7 in [1]) that the operator

$$
\mathcal{O}\left(U, Y^{\prime}\right) \stackrel{z-S^{\prime}}{\longrightarrow} \mathcal{O}\left(U, Y^{\prime}\right)
$$

is injective with closed range for each open set $U \subset \rho_{\mathrm{e}}(T)$. To complete the proof, it suffices to apply part (a) of Corollary 3.6.

Corollary 3.8. Let $T \in L(X)$ and $S \in L(Y)$ be continuous linear operators on complex Banach spaces $X$ and $Y$. Let $A \in L(X, Y)$ and $B \in L(Y, X)$ be operators with $S A=A T$ and $T B=B S$.

(a) If $A$ and $B$ have dense range, and if $S$ and $T$ satisfy property $(\beta)$, then

$$
\sigma(S)=\sigma(T) \quad \text { and } \quad \sigma_{\mathrm{e}}(S)=\sigma_{\mathrm{e}}(T)
$$


(b) If $A$ and $B$ are injective, and if $S$ and $T$ satisfy property ( $\delta$ ), then

$$
\sigma(S)=\sigma(T) \quad \text { and } \quad \sigma_{\mathrm{e}}(S)=\sigma_{\mathrm{e}}(T)
$$

(c) Suppose that $S$ and $T$ are quasisimilar. If $T$ satisfies property $(\beta)$ or property $(\delta)$, and if $S$ satisfies property $(\beta)$ or property $(\delta)$, then

$$
\sigma(S)=\sigma(T) \quad \text { and } \quad \sigma_{\mathrm{e}}(S)=\sigma_{\mathrm{e}}(T) .
$$

Part (a) of Corollary 3.8 was proved by Yang [14] for Hilbert-space operators with empty point spectrum, and by Putinar [12] in the general case. Part (b) was proved, with different methods, by Miller and Miller in [11].

Corollary 3.9. Let $T \in L(X)$ be a continuous linear operator on a complex Banach space. Suppose that $T$ satisfies the single-valued extension property. Then $T$ satisfies property $(\beta)$ on $\rho_{\mathrm{e}}(T)$. If $T \in L(X)$ and $T^{\prime} \in L\left(X^{\prime}\right)$ both satisfy the single-valued extension property, then $T$ is decomposable modulo $\sigma_{\mathrm{e}}(T)$.

Proof. The first part is an obvious consequence of Theorem 3.1. Suppose that both $T$ and $T^{\prime}$ satisfy the single-valued extension property. Then both $T$ and $T^{\prime}$ satisfy property $(\beta)$ on $\rho_{\mathrm{e}}(T)$ by Theorem 3.1. Hence, $T$ satisfies property $(\beta)$ and property $(\delta)$ on $\rho_{\mathrm{e}}(T)$, and, thus, is decomposable modulo $\sigma_{\mathrm{e}}(T)$ (see [1, Theorems 2.1 and 3.4]).

\section{Essential spectra of quasisimilar operators}

In [7], Herrero proved that, for any pair of quasisimilar Banach space operators $T \in L(X)$ and $S \in L(Y)$, each component of $\sigma_{\mathrm{e}}(T)$ meets $\sigma_{\mathrm{e}}(S)$, and vice versa. The main ingredient of the original proof was Herrero's result (see [7, Theorem 2]) on the Fredholm domain of multicyclic operators. In the following we indicate how the results of the previous section can be used to give an alternative proof.

Proposition 4.1. Let $S \in L(X)$ be a continuous linear operator on a complex Banach space $X$, and let $\Omega$ be a bounded open set in $\mathbb{C}$ such that

$$
\sigma=\Omega \cap \sigma_{\mathrm{e}}(S)
$$

is compact. Suppose that $U$ is an open set with $\partial \Omega \subset U \subset \mathbb{C} \backslash \sigma$.

(a) If $S$ satisfies the single-valued extension property on $\Omega \cap U$, then the map

$$
\mathcal{O}(\Omega, X) \stackrel{z-S}{\longrightarrow} \mathcal{O}(\Omega, X)
$$

is injective with closed range.

(b) If $S^{\prime}$ satisfies the single-valued extension property on $\Omega \cap U$, then the map

$$
\mathcal{O}\left(\Omega, X^{\prime}\right) \stackrel{z-S^{\prime}}{\longrightarrow} \mathcal{O}\left(\Omega, X^{\prime}\right)
$$

is injective with $w^{*}$-closed range. 
Proof. Under the hypothesis of part (a), let $\left(f_{n}\right)$ be a sequence in $\mathcal{O}(\Omega, X)$ such that $\lim _{n \rightarrow \infty}(z-S) f_{n}=0$. For any closed $\operatorname{disc} K$ in $\Omega$, we can choose a connected open set $G$ with $K \subset G \subset \subset \Omega$ and $\partial G \subset U$. By the maximum modulus principle and Theorem 3.1,

$$
\left\|f_{n}\right\|_{\infty, K} \leqslant\left\|f_{n}\right\|_{\infty, \partial G} \stackrel{n}{\rightarrow} 0 .
$$

Secondly, suppose that $S$ is as in part (b). Let $\Omega_{0} \subset \subset \Omega$ be open with $\Omega \cap(\mathbb{C} \backslash U) \subset$ $\Omega_{0}$. Set $\Omega_{1}=\mathbb{C} \backslash \bar{\Omega}_{0}$. The disjoint union $V=\Omega_{0} \cup \Omega_{1}$ is open and contains the set $\Sigma=\mathbb{C} \backslash(U \cap \Omega)$. By Theorem 3.1, the operator $S^{\prime}$ satisfies property $(\beta)$ modulo $\Sigma$. According to Lemma 6 and Theorem 21 in [1], the operator

$$
\mathcal{O}(V)^{\prime} \hat{\otimes} X \stackrel{z-S}{\longrightarrow} \mathcal{O}(V)^{\prime} \hat{\otimes} X
$$

is surjective. The same holds with $V$ replaced by $\Omega_{0}$. Using Köthe-Grothendieck duality, one obtains that the space $\mathcal{O}\left(\Omega_{0}\right)^{\prime} \hat{\otimes} X$ is an $(L F)$-space (cf. the proof of Theorem 5 in [1]). By the open mapping principle for $(L F)$-spaces (see $[9, \S 34.8(4)]$ ), and by general duality theory (see $[9, \S 32.3(4)]$ ), the map

$$
\mathcal{O}\left(\Omega_{0}, X^{\prime}\right) \stackrel{z-S^{\prime}}{\longrightarrow} \mathcal{O}\left(\Omega_{0}, X^{\prime}\right)
$$

is injective with $w^{*}$-closed range.

By applying this observation to each set $\Omega_{n}$ in a suitably chosen exhaustion $\left(\Omega_{n}\right)$ of $\Omega$, the proof of part (b) is completed.

Elementary examples show that the assertions of the last proposition become wrong without the hypothesis on the single-valued extension property near the boundary of $\Omega$. Even for open sets $\Omega$ containing the whole essential spectrum $\sigma_{\mathrm{e}}(T)$ of a given operator $T \in L(X)$, one cannot expect that $(z-T) \mathcal{O}(\Omega, X)$ is a closed subset of $\mathcal{O}(\Omega, X)$. For example, let $X=\ell^{2}$, and let $T \in L(X)$ be the unilateral backward shift on $X$. Set $\Omega=\{z \in \mathbb{C} ; 0<|z|<2\}$. Then the space

$$
X_{T}(0)=\{x \in X ; x \in(z-T) \mathcal{O}(\Omega, X)\}=\left\{x \in X ; \lim _{n \rightarrow \infty}\left\|T^{n} x\right\|^{1 / n}=0\right\}
$$

is a proper dense subspace of $X$. Hence, $(z-T) \mathcal{O}(\Omega, X)$ cannot be closed in $\mathcal{O}(\Omega, X)$.

Let $T \in L(X)$ be a continuous linear operator on a complex Banach space $X$, and let $U$ be an open set in $\mathbb{C}$ such that

$$
\mathcal{O}(U, X) \stackrel{z-T}{\longrightarrow} \mathcal{O}(U, X)
$$

is injective with closed range. If the quotient space $\mathcal{O}(U, X) /(z-T) \mathcal{O}(U, X)$ is nuclear, then the induced map

$$
\mathcal{O}\left(U, X^{q}\right) \cong \mathcal{O}(U, X)^{q} \stackrel{z-T^{q}}{\longrightarrow} \mathcal{O}(U, X)^{q} \cong \mathcal{O}\left(U, X^{q}\right)
$$

is a topological isomorphism (see the preliminaries), and, therefore,

$$
U \subset \mathbb{C} \backslash \sigma\left(T^{q}\right)=\mathbb{C} \backslash \sigma_{\mathrm{e}}(T) \quad(\text { cf. }[3, \text { p. 12]) }
$$


Let $T \in L(X)$ and $S \in L(Y)$ be quasisimilar operators on complex Banach spaces $X$ and $Y$. Let us assume that there is a component $\sigma_{0}$ of $\sigma_{\mathrm{e}}(S)$ with $\sigma_{0} \cap \sigma_{\mathrm{e}}(T)=\emptyset$. Since $\sigma_{\mathrm{e}}(S)$ is compact, the set $\sigma_{0}$ is the intersection of all closed and open subsets $\sigma$ of $\sigma_{\mathrm{e}}(S)$ containing $\sigma_{0}$. Hence, there is a closed and open subset $\sigma$ of $\sigma_{\mathrm{e}}(S)$ with $\sigma_{0} \subset \sigma \subset \rho_{\mathrm{e}}(T)$. We fix an open set $\Omega$ in $\mathbb{C}$ with

$$
\sigma \subset \Omega \subset \subset \rho_{\mathrm{e}}(T) \cap\left(\mathbb{C} \backslash\left(\sigma_{\mathrm{e}}(S) \backslash \sigma\right)\right)
$$

To prove the result of Herrero cited above, we have to produce a contradiction. Elementary arguments from Fredholm theory can be used, as in Herrero's original proof, to reduce the general case to the two main cases

$$
\operatorname{Ker}(\lambda-S)=0, \quad \text { for all } \lambda \in \partial \Omega
$$

or

$$
\operatorname{Ker}\left(\lambda-S^{\prime}\right)=0, \quad \text { for all } \lambda \in \partial \Omega \text {. }
$$

In the first case, part (a) of Proposition 4.1 and the first part of Corollary 3.6 imply that there is a topological isomorphism

$$
\mathcal{O}(\Omega, X) /(z-T) \mathcal{O}(\Omega, X) \stackrel{\sim}{\longrightarrow} \mathcal{O}(\Omega, Y) /(z-S) \mathcal{O}(\Omega, Y) .
$$

In the second case, the remaining parts of Proposition 4.1 and Corollary 3.6 yield a topological isomorphism

$$
\mathcal{O}\left(\Omega, X^{\prime}\right) /\left(z-T^{\prime}\right) \mathcal{O}\left(\Omega, X^{\prime}\right) \stackrel{\sim}{\longrightarrow} \mathcal{O}\left(\Omega, Y^{\prime}\right) /\left(z-S^{\prime}\right) \mathcal{O}\left(\Omega, Y^{\prime}\right) .
$$

In both cases, the space on the right is a nuclear Fréchet space, and, hence, $\Omega \cap \sigma_{\mathrm{e}}(S)=\emptyset$.

Theorem 4.2 (Herrero). Let $T \in L(X)$ and $S \in L(Y)$ be quasisimilar operators on complex Banach spaces $X$ and $Y$. Then each component of $\sigma_{\mathrm{e}}(S)$ meets $\sigma_{\mathrm{e}}(T)$, and vice versa.

Proof. For the sake of completeness we indicate how to reduce the general case to the two special cases considered above.

Let us choose the closed and open subset $\sigma$ of $\sigma_{\mathrm{e}}(S)$ and its open neighbourhood $\Omega$ exactly as explained above. Since the discontinuity points of the function

$$
\rho_{\mathrm{e}}(S) \rightarrow \mathbb{C}, \quad z \mapsto \operatorname{dim} \operatorname{Ker}(z-S)
$$

form a discrete subset of $\rho_{e}(S)$ (see [3, Remark 9.4.6]), one can achieve, by shrinking $\Omega$ if necessary, that none of these discontinuity points belongs to $\partial \Omega$. Since one can always replace $\Omega$ by $\operatorname{Int}(\bar{\Omega})$, we may suppose in addition that $\partial \Omega$ has no isolated points.

The spaces

$$
M=\bigvee_{\lambda \in \partial \Omega} \operatorname{Ker}(\lambda-T) \text { and } N=\bigvee_{\lambda \in \partial \Omega} \operatorname{Ker}(\lambda-S)
$$


are closed invariant subspaces for $T$ and $S$, respectively. Since, for $\mu \in \mathbb{C} \backslash \partial \Omega$ and for $\lambda \in \partial \Omega$, the operators

$$
\mu-T: \operatorname{Ker}(\lambda-T) \rightarrow \operatorname{Ker}(\lambda-T), \quad(\mu-S): \operatorname{Ker}(\lambda-S) \rightarrow \operatorname{Ker}(\lambda-S)
$$

are bijective, the subspaces $(\mu-T) M \subset M$ and $(\mu-S) N \subset N$ are dense subspaces. For $\mu \in \rho_{\mathrm{e}}(T)$, the space $(M+\operatorname{Ker}(\mu-T)) / \operatorname{Ker}(\mu-T)$ is a closed subspace of the quotient space $X / \operatorname{Ker}(\mu-T)$. Hence, $(\mu-T) M \subset M$ is closed. By applying the same argument to $S$, we obtain that

$$
\Omega \cap \sigma_{\mathrm{e}}(T \mid M)=\emptyset, \quad \Omega \cap \sigma_{\mathrm{e}}(S \mid N) \subset \sigma
$$

and that the operators $\lambda-S: N \rightarrow N(\lambda \in \Omega \backslash \sigma)$ are surjective. It follows from part (b) of Proposition 4.1 and part (b) of Corollary 3.6 that

$$
\mathcal{O}\left(\Omega, M^{\prime}\right) /\left(z-(T \mid M)^{\prime}\right) \mathcal{O}\left(\Omega, M^{\prime}\right) \cong \mathcal{O}\left(\Omega, N^{\prime}\right) /\left(z-(S \mid N)^{\prime}\right) \mathcal{O}\left(\Omega, N^{\prime}\right)
$$

are topologically isomorphic. Therefore, $\Omega \subset \rho_{\mathrm{e}}(S \mid N)$.

We complete the proof by showing that $\Omega \subset \rho_{\mathrm{e}}(S / N)$. For $\mu \in \partial \Omega$, the operator $\mu-(S / N) \in L(Y / N)$ has finite-codimensional range, and, hence,

$$
\mu-(S / N)^{q} \in L\left((Y / N)^{q}\right)
$$

is surjective (Theorem 2.6.4 in [3]). Since $\Omega \backslash \sigma \subset \rho_{\mathrm{e}}(S / N)=\rho\left((S / N)^{q}\right)$, it follows that $\partial \Omega \subset \rho_{\mathrm{e}}((S / N))$. We claim that $\partial \Omega \cap \sigma_{p}(S / N)=\emptyset$. In view of the definition of the space $N$, it suffices to show that the operators

$$
\mu-S: N \rightarrow N \quad(\mu \in \partial \Omega)
$$

are surjective. Fix a point $\mu \in \partial \Omega$. Let $C$ and $C_{N}$ be the components of $\mu$ in $\rho_{\mathrm{e}}(S)$ and $\rho_{\mathrm{e}}(S \mid N)$, respectively. Let $\left(\mu_{n}\right)$ be a sequence in $C \cap C_{N} \cap(\partial \Omega \backslash\{\mu\})$ with $\lim _{n \rightarrow \infty} \mu_{n}=\mu$. Since $\mu_{n}, \mu \in C \cap \partial \Omega$, we have

$$
\operatorname{dim} \operatorname{Ker}\left(\mu_{n}-S \mid N\right)=\operatorname{dim} \operatorname{Ker}\left(\mu_{n}-S\right)=\operatorname{dim} \operatorname{Ker}(\mu-S)=\operatorname{dim} \operatorname{Ker}(\mu-S \mid N),
$$

for all $n$. Since $\mu_{n}, \mu \in C_{N}$, we have

$$
\operatorname{dim}\left(N /\left(\mu_{n}-S\right) N\right)=\operatorname{dim}(N /(\mu-S) N),
$$

for all $n$. By Remark 9.4.6 in [3], the set of discontinuity points of the function

$$
C_{N} \rightarrow \mathbb{Z}, \quad z \mapsto \operatorname{dim}(N /(z-S) N)
$$

is discrete in $C_{N}$. Therefore, $(\mu-S) N=N$.

Thus, we have shown that $(\partial \Omega) \cap\left(\sigma_{p}(S / N) \cup \sigma_{\mathrm{e}}(S / N)\right)=\emptyset$. By the first parts of Corollary 3.6 and Proposition 4.1, the spaces

$$
\mathcal{O}(\Omega, X / M) /(z-T / M) \mathcal{O}(\Omega, X / M) \cong \mathcal{O}(\Omega, Y / N) /(z-S / N) \mathcal{O}(\Omega, Y / N)
$$

are topologically isomorphic nuclear Fréchet spaces. Therefore, $\Omega \subset \rho_{\mathrm{e}}(S / N)$, and the proof is complete. 
As a final application we also indicate that the result originally used by Herrero to prove Theorem 4.2 (see [7, Theorem 2]) can be obtained as an elementary consequence of the methods from $\S 3$.

Let $n \geqslant 1$ be a natural number. Recall that an operator $T \in L(X)$ on a complex Banach space $X$ is $n$-multicyclic if there are vectors $y_{1}, \ldots, y_{n} \in X$ such that

$$
X=\bigvee\left\{p(T) y_{i} ; p \in \mathbb{C}[z] \text { and } i=1, \ldots, n\right\}
$$

and if, moreover, $n$ is the minimal natural number with this property. In this case, for $z \in \rho_{\mathrm{e}}(T)$

$$
X=\bigvee\left\{p(T) y_{i} ; p \in \mathbb{C}[z], i=1, \ldots, n\right\}=(z-T) X+\bigvee\left\{y_{1}, \ldots, y_{n}\right\}
$$

In particular, $-\operatorname{ind}(z-T) \leqslant \operatorname{dim}(X /(z-T) X) \leqslant n$. In [6], Herrero proved the following result on the structure of the Fredholm domain of $n$-multicyclic operators.

Theorem 4.3. Let $T \in L(X)$ be $n$-multicyclic, and let $\Omega$ be a component of $\rho_{\mathrm{e}}(T)$ with ind $(z-T)=-n$ for $z \in \Omega$. Then $\Omega$ is simply connected.

Proof. Note that, for $z \in \Omega$, the elements $\left[y_{1}\right], \ldots,\left[y_{n}\right]$ form a basis for $X /(z-T) X$, and that $\operatorname{Ker}(z-T)=\{0\}$. Hence (with the notation from the proof of Lemma 3.2), each of the operators

$$
d^{1}(z): X \oplus \mathbb{C}^{n} \rightarrow X, \quad\left(x,\left(t_{i}\right)\right) \mapsto(z-T) x-\sum_{i=1}^{n} t_{i} y_{i} \quad(z \in \Omega)
$$

is a topological isomorphism. Observing that in each step of the proof of Lemma 3.2 one can choose $V=\Omega$, we obtain that the map

$$
h^{1}: \mathcal{O}(\Omega)^{n} \rightarrow \mathcal{O}(\Omega, X) /(z-T) \mathcal{O}(\Omega, X), \quad\left(h_{i}\right) \mapsto\left[\sum_{i=1}^{n} h_{i} y_{i}\right]
$$

is a vector-space isomorphism with inverse induced by

$$
k^{1}: \Omega \rightarrow L\left(X, \mathbb{C}^{n}\right), \quad k^{1}(z)=-\pi_{\mathbb{C}^{n}} \circ d^{1}(z)^{-1} .
$$

The composite $J: X \rightarrow \mathcal{O}(\Omega)^{n}$ of the two operators

$$
\begin{gathered}
X \rightarrow \mathcal{O}(\Omega, X) /(z-T) \mathcal{O}(\Omega, X), \quad x \mapsto[x] \\
\mathcal{O}(\Omega, X) /(z-T) \mathcal{O}(\Omega, X) \rightarrow \mathcal{O}(\Omega)^{n}, \quad[f] \mapsto k^{1} f
\end{gathered}
$$

satisfies, for any system of polynomials $p_{1}, \ldots, p_{n} \in \mathbb{C}[z]$,

$$
J\left(\sum_{i=1}^{n} p_{i}(T) y_{i}\right)=\left(p_{i}\right)_{i=1}^{n}
$$

Fix a compact subset $L$ of $\Omega$ and a bounded component $H$ of $\mathbb{C} \backslash L$. It suffices to show that the compact set $\sigma=H \cap \sigma_{\mathrm{e}}(T)$ is empty. Choose a cycle $\gamma$ surrounding $\sigma$ in $H$ such 
that $\gamma$ is contained in $\Omega$, and fix a family of left inverses $R(z)(z \in \Omega)$ of $z-T$ depending continuously on $z$ (e.g. $R(z)=\pi_{X} \circ d^{1}(z)^{-1}$ ). Since $\mathcal{O}(\Omega)^{n}$ is nuclear, the operator

$$
K: \mathcal{O}(\Omega)^{n} \rightarrow X, \quad\left(f_{i}\right)_{i=1}^{n} \mapsto \sum_{i=1}^{n} \frac{1}{2 \pi \mathrm{i}} \int_{\gamma} f_{i}(z) R(z) y_{i} \mathrm{~d} z
$$

is nuclear, and, hence, compact. For $i=1, \ldots, n$ and $p \in \mathbb{C}[z]$,

$$
(K J)\left(p(T) y_{i}\right)=\frac{1}{2 \pi \mathrm{i}} \int_{\gamma} p(z) R(z) y_{i} \mathrm{~d} z=\left(\frac{1}{2 \pi \mathrm{i}} \int_{\gamma} R(z) \mathrm{d} z\right)\left(p(T) y_{i}\right)
$$

But the operator

$$
\left(\frac{1}{2 \pi \mathrm{i}} \int_{\gamma} R(z)^{q} \mathrm{~d} z\right)=(K J)^{q}=0
$$

is precisely the spectral projection corresponding to the closed and open subset $\sigma$ of $\sigma\left(T^{q}\right)=\sigma_{\mathrm{e}}(T)$. Therefore, $\sigma=\emptyset$, and the proof is complete.

\section{References}

1. E. Albrecht and J. Eschmeier, Analytic functional models and local spectral theory, Proc. Lond. Math. Soc. 75 (1997), 323-348.

2. J. EsCHMEIER, Analytische Dualität und Tensorprodukte in der mehrdimensionalen Spektraltheorie, Schriftenreihe des Math. Inst. der Univ. Münster 42 (1987), 132pp.

3. J. EschmeIER AND M. PUTINAR, Spectral decompositions and analytic sheaves, London Mathematical Society Monographs, vol. 10 (Clarendon Press, Oxford, 1996).

4. J. K. FINCH, The single-valued extension property on a Banach space, Pacific J. Math. 58 (1975), 61-69.

5. H. Grauert and R. Remmert, Theory of Stein spaces (Springer, Berlin, 1979).

6. D. HERRERO, The Fredholm structure of an $n$-multicyclic operator, Indiana Univ. Math. J. 36 (1987), 549-566.

7. D. Herrero, On the essential spectra of quasisimilar operators, Can. J. Math. 40 (1988), 1436-1457.

8. G. KÖTHE, Topological vector spaces I (Springer, Berlin, 1969).

9. G. KöTHE, Topological vector spaces II (Springer, New York, 1979).

10. R. Kultze, Garbentheorie (Teubner, Stuttgart, 1970).

11. T. L. Miller AND V. G. MiLler, Equality of essential spectra of quasisimilar operators with property $(\delta)$, Glasgow Math. J. 38 (1996), 21-28.

12. M. PUTINAR, Quasisimilarity of tuples with Bishop's property $(\beta)$, Integral Eqn. Operator Theory 15 (1992), 1047-1052.

13. H. SCHAEFER, Topological vector spaces (Macmillan, New York, 1966).

14. L. YANG, Quasisimilarity of hyponormal and subdecomposable operators, J. Funct. Analysis 112 (1993), 204-217. 